\title{
COSMOS: PEER-TO-PEER COLLABORATIVE STREAMING AMONG MOBILES *
}

\author{
Man-Fung Leung ${ }^{\dagger} \quad$ S.-H. GaryChan ${ }^{\dagger} \quad$ Oscar C. Au ${ }^{\ddagger}$ \\ ${ }^{\dagger}$ Department of Computer Science ${ }^{\ddagger}$ Department of Electrical and Electronic Engineering \\ The Hong Kong University of Science and Technology \\ Clear Water Bay, Kowloon, Hong Kong SAR \\ \{csfung, gchan, eeau\}@ust.hk
}

\begin{abstract}
In traditional mobile streaming networks such as $3 \mathrm{G}$ cellular networks, all users pull streams from a server. Such pull model leads to high streaming cost and problem in system scalability. In this paper, we propose and investigate a scalable and cost-effective protocol to distribute multimedia content to mobiles in a peer-to-peer manner. Our protocol, termed Collaborative Streaming among Mobiles (COSMOS), makes use of multiple description coding (MDC) and data sharing to achieve high performance. In COSMOS, only a few peers pull video descriptions through a telecommunication channel. Using a free broadcast channel (such as Wi-Fi and bluetooth), they share the descriptions to nearby neighbors in an ad-hoc manner. This way reduces greatly the telecommunication cost and cellular bandwidth requirement. As video descriptions are supplied by multiple peers, COSMOS is robust to peer failure. Since broadcasting is used to distribute video data, the protocol is highly scalable to large number of users. By taking turns to pull descriptions, we show through simulation that peers can effectively share, and hence substantially reduce, streaming cost. As peers can often obtain a number of descriptions from nearby neighbors, they enjoy lower delay as compared to a recent scheme CHUM.
\end{abstract}

\section{INTRODUCTION}

In traditional mobile streaming system such as $3 \mathrm{G}$ cellular network, users in the range of a base station "pull" streams from a remote server. ${ }^{1}$ Depending on the amount of data streamed, they are charged for certain streaming fee. As cellular channels are precious and limited in number, such approach is usually costly and is not scalable in terms of user capacity. In addition, users have to be in the coverage of the base station in order to be served. This greatly limits the pervasive deployment of multimedia streaming services.

With the evolution of mobile technology, we have witnessed great improvement in processing capability, battery power, and memory of mobile devices such as cellular phones and PDAs. Many mobile devices nowadays can already connect to each other and exchange data using some secondary wireless channels such as IEEE 802.11 or Bluetooth which can often be turned on simultaneously with the cellular channel $[1,2]$. As these channels are broadcasting and free in nature, we can make use of them to achieve cost-effective collaborative peer-to-peer streaming.

${ }^{*}$ This work is supported, in part, by Direct Allocation Grant (DAG05/06.EG10) and Innovation and Technology Commission (ITS/122/03 and GHP/045/05) of the Hong Kong Special Administrative Region, China.

${ }^{1}$ In this paper, we use "peers", "mobiles", "nodes", and "users" interchangeably.

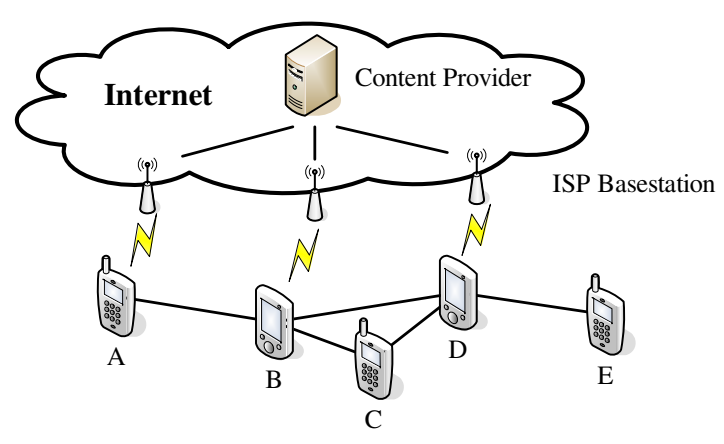

Fig. 1. Mobile devices in COSMOS network cooperatively download and share video descriptions pulled from a content provider.

In this paper, we propose and study a scalable and cost-effective protocol called Collaborative Streaming among Mobiles (COSMOS) to share multimedia data. ${ }^{2}$ We make use of the secondary free channels already available in mobile devices today to form a wireless mobile network. The mobiles share their multimedia streams among their peers in near vicinity. In COSMOS, some mobiles are "pullers," who pull streams from the base station and hence are the payers in the system. By taking turns in being the pullers, the mobiles can more fairly and cost-effectively share streaming contents. Such approach is particularly attractive for popular multimedia streams such as live sport events and TV news, which may be of interest to many users within the vicinity of each other.

COSMOS may be incorporated with multiple description coding (MDC), where a video stream is coded into multiple independent "descriptions" which may be arbitrarily combined and played back. The more descriptions one receives, the higher the video quality is. With MDC, a peer in COSMOS randomly selects and downloads a video description through a telecommunication link (the cellular channel) and broadcasts to its nearby neighbors with a certain broadcast scope or hop using the secondary wireless channel. In this way, a peer may collect more than one description while paying only for one description. A peer stops pulling description if it receives full set of descriptions from the broadcast channel. In COSMOS, we hence have two types of mobiles at any instant of time: pullers who pay for their streams, and passive receivers who do not need to pay at that time. As opposed to the traditional "what-you-pull-is-what-you-get" approach, our system is much more scalable with its performance (in

${ }^{2}$ We focus on only the technical details on sharing mechanism of COSMOS here. Issues such as payment security and authentication, right management, and attacks are beyond the scope of this paper. 
terms of delay, cost, fault resilience, etc.) improves, rather than decreases, with the number of peers.

Figure 1 illustrates our COSMOS system, where five mobile devices labeled from $A$ to $E$ form a wireless network using their secondary channel. Mobiles $A, B$, and $D$ pull different video descriptions from the content provider. They relay the multimedia data received to others by rebroadcasting using the secondary channel, hence providing service to $C$ and $E$. Note that some nodes $(C$ and $E$ in the figure) may not need to download data, the total telecommunication streaming cost can be reduced. In the figure, assuming that the broadcast scope (hop) is one, $A, B$, and $D$ are pullers paying one description and enjoying two, three and two descriptions, respectively, while $C$ and $E$ are passive receivers enjoying two and one description, respectively.

Clearly, COSMOS enjoys the following strengths:

- Reduction in streaming cost and bandwidth requirement per node: Because peer needs to pull only a video description instead of the full video stream (which may be of much higher bandwidth), COSMOS achieves much lower cellular bandwidth requirement and streaming cost given a certain group of users.

- Low delay: The delay depends on the broadcast hop. In general, one to two broadcast hops would be enough, and hence the delay is low in our system.

- High scalability: COSMOS is a fully distributed protocol. It is simple to implement, and each mobile has little processing and transmission overhead in terms of control messaging and membership maintenance. As a single broadcast may cover a large number of users in the coverage range without the need of any mobile forwarding, it is scalable to large group.

- Robustness to peer dynamics and failure: Each mobile may receive multiple descriptions at the same time. Therefore, the departure or failure of a node would not break the video continuity. Furthermore, some peers may enjoy replicated description depending on their locations in the network. Therefore, if some pullers of that description fails, these peers would not be affected. This MDC feature and intrinsic redundancy greatly improves the robustness of the system.

In this paper, we address and study the following important issues in COSMOS. The first one is how peers pull different video descriptions in a distributed manner so that good video quality can be achieved and the wireless channel can be better utilized. The second issue is the protocol on how peers take turns in pulling descriptions to achieve better fairness in cost sharing. Lastly, we address how to handle network dynamic in the presence of user joining, leaving, and failures. We have conducted simulation to evaluate the performance of COSMOS and compare it with a recently proposed system, CHUM [3]. Our results show that COSMOS achieves better performance in delay, cost fairness, video bitrate achieved, and resilience to peer failure, with some sacrifice in cost.

We briefly discuss previous work as follows. COSMOS may use MDC, which has been extensively studied before (See, for example, $[4,5])$. Using a secondary channel for mobile data delivery has been investigated in iCAR, which integrates cellular system with ad-hoc network [6]. However, the previous work focuses mainly on how to relay data from a mobile to the base station, while we discuss in COSMOS how to share data collaboratively among the mobile hosts to reduce telecommunication cost. While there is no fault issue in iCAR (as the relay points are stationary and reliable), we need to address user dynamics and fault issues.

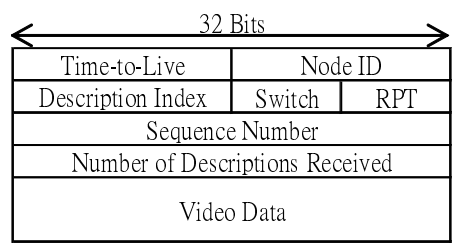

Fig. 2. Format of video packet broadcasted.

A more recent work related to ours is CHUM, which shares multimedia data among mobile devices in an ad-hoc manner [3]. Similar to COSMOS, some peers in CHUM pull multimedia content and share it to their peers. While CHUM is based on a tree topology structure for data forwarding using 2-hop knowledge, COSMOS is based on a mesh topology with broadcast. Therefore, COSMOS has lower delay, lower bandwidth requirement, better fault resilience, and lower processing and maintenance overhead at nodes. WIANI is a multi-hop WLAN which makes use of ad-hoc channel for coverage extension [7]. While one of the major issues in WIANI is how to do ad-hoc routing, COSMOS routing is simpler as it is based on broadcasting and mobiles do not need to know the addresses of each other (no point-to-point communication is necessary). Furthermore, as opposed to WIANI, COSMOS mobiles use a separate channel for peer-to-peer sharing.

The rest of the paper is organized as follows. We first present the details of COSMOS protocol in Section 2. We then present our illustrative simulation results and the comparison with CHUM in Section 3. In Section 4, we conclude with some future work.

\section{DESCRIPTION OF COSMOS}

We present the details of COSMOS protocol, in regards to how a peer selects and broadcasts its video descriptions in section 2.1. In section 2.2, we introduce how peers take turns to pull video data from the content provider and how to handle peer dynamics and failures.

\subsection{Video distribution}

In a video server, the video is encoded into $D$ independent video descriptions using MDC. A peer randomly chooses and pulls a description through the cellular link. The single description allows the peer to have some basic quality. To improve its video quality, it may further pull more descriptions from the content provider or be helped from its neighbors.

In COSMOS, peers can collect more video descriptions through the broadcast channel. Pullers broadcast the video data they pull to their neighbors with a certain broadcast scope. As the delay increases with the broastcast scope, the scope should not be too high Figure 2 shows the format of the video packet broadcasted among peers. Before broadcasting the video packet, pullers set the Timeto-Live to $S$ (which is the broadcast scope or hop). When a peer receives the packet, it decrements the Time-to-Live by 1 . Peers rebroadcast the packet if the TTL value is greater than 0 .

Since peers randomly select video descriptions to pull, it may happen that two mobiles pull and broadcast the same description to each other. Though this redundancy leads to some failure resilience, it increases streaming cost and cellular bandwidth requirement. It is better for one of them to pull another description to improve the video quality and bandwidth utilization. In general, we would like 
the node with more neighbors to be the puller, as its description shared is beneficial to more users. Since the number of video descriptions a peer received somewhat indicates its number of nearby neighbors, the peer receiving more descriptions would keep sharing its pulled description. The Number of Description Received field in the video packet helps multiple potential pullers to resolve whom the puller should be. In case of a tie on the number of descriptions, the peer with the largest Node ID, which is randomly generated as a peer joins, would pull the description. The peers without the full video descriptions would randomly choose an unavailable description to pull. If the peer finds that all descriptions are already available from the broadcast secondary channel, it becomes a passive receiver.

\subsection{Cost sharing and group dynamics}

As the number of peers increases, some may not need to pull any descriptions. In order to more fairly distribute the load and the telecommunication cost among the peers, COSMOS has a mechanism to exchange the roles between pullers and passive receivers when a puller has been downloading video data for some time.

A certain time $T$ seconds before a puller would like to stop pulling, it sets the Switch flag of the video packet and $R P T$ (remaining pull-time) as $T$ to inform other peers on its intention of role switching. The packet is broadcasted with the same scope $S$. A passive receiver who receives the video packet with Switch flag set starts a random timer with maximum time $T$. If by this time it does not receive the corresponding description from its neighbors, it becomes a puller of the description by rebroadcasting the description received. In other words, the one with the earliest timer becomes the pullers.

Before a puller leaves the network, it notifies other peers so that they can contend to pull the description for sharing. A leaving puller sets the Switch flag of the video packet and RPT to 0. In this case, the other peers then start a random timer of maximum value $L$. What follows is similar to the role switching mechanism.

COSMOS is robust to peer failure since some descriptions may be duplicated. If a puller fails, the same description can be supplied from other peers. Peers can buffer and order the video packets received according to their Description Index and Sequence Number fields, which are the identity number of description and time stamp of the video, respectively. In this way, duplicate packets can be identified and removed. Peers need to keep track of video descriptions received from other peers. If a peer finds that some of its video descriptions have been missing for a time (due to, for example, node failures), it starts pulling and sharing the video description after a certain random timer $L$.

\section{ILLUSTRATIVE SIMULATION RESULTS}

In this section, we describe the simulation used to compare the performances of COSMOS and CHUM. Peers are randomly placed on a 100 units $\times 100$ units area and their coverage range is 15 units. Peers enter the system with Poisson arrival rate $\lambda$ (request/unit time). Each peer remains in the system with exponential time of mean $1 / \mu$ if it does not fail. A peer may fail with rate $f$ (request/unit time). Therefore, at steady state, the average number of peers in the system is $N=\lambda /(\mu+f)$ and the probability that a peer fails is $f /(\mu+f)$. In this paper, we normalized the time such that $\mu=1$ time unit.

For COSMOS, we consider that bandwidth is normalized such that for $D$ descriptions, each description is of bandwidth $1 / D$ while a full stream is of bandwidth 1 . We have implemented an eventdriven simulation to study the system. All data are taken at steady state.

\subsection{Metrics}

Consider peer $i$ with its total time $T_{i}$ in system. Let $t$ be an instant of its lifetime in the system and $N$ be the number of peers examined. We consider the following performance metrics in our study:

1. Delay: This is the minimum number of broadcasts by other peers before a peer receives a packet for a particular description. Let $H_{i}(t)$ be maximum delay of all its descriptions received at time $t$. We are interested in the average delay defined by $\sum_{i} \int_{T_{i}} H_{i}(t) d t / \sum_{i} T_{i}$.

2. Cost: Cost refers to the streaming cost per unit time for a user. Let $P_{i}(t)$ be the total description bandwidth peer $i$ pulls at time $t$. We are interested in the average cost over all users defined as $\sum_{i} \int_{T_{i}} P_{i}(t) d t / \sum_{i} T_{i}$.

3. Cost Variation: We define streaming cost per unit time for peer $i$ as $C_{i}=\int_{T_{i}} P_{i}(t) d t / T_{i}$. Let $\sigma_{C}$ and $\mu_{C}$ be the standard deviation and the mean of $C_{i}$, respectively. We define cost variation as the coefficient of variation given by $\sigma_{C} / \mu_{C}$.

4. Video Bitrate: This refers to the effective description bandwidth received (except duplication) per unit time for a peer. Let $R_{i}(t)$ be the effective description bandwidth peer $i$ receives at time $t$. We are interested in the average video bitrate defined as $\sum_{i} \int_{T_{i}} R_{i}(t) d t / \sum_{i} T_{i}$.

5. Bitrate Fluctuation: Let $\sigma_{R_{i}}$ and $\mu_{R_{i}}$ be the standard deviation and the mean of $R_{i}(t)$, respectively. We define bitrate fluctuation of peer $i$ as the coefficient of variation given by $\sigma_{R_{i}} / \mu_{R_{i}}$. We are interested in the average bitrate fluctuation over all users, i.e. $\sum_{i}\left(\sigma_{R_{i}} / \mu_{R_{i}}\right) / N$.

Unless otherwise stated, we set $f=0.1$ (request/unit time), $\lambda=100$ (request/unit time), and number of descriptions $D=4$ as baseline parameters. We consider two broadcast scopes for COSMOS, with $S=1$ and $S=2$.

\subsection{Experiments}

Figure 3(a) compares that the performance of COSMOS with CHUM in terms of delay as $N$ increases. Clearly, COSMOS has a much lower delay. In COSMOS, the video packets are broadcasted within the scope $S$ and hence delay is limited. For CHUM, the video data is forwarded until it reaches leaf nodes of tree. When the network is small, its average tree height and the average delay increases with $N$. However, average delay drops slightly beyond a certain value. This is because when the area becomes very crowded, the average path length (in term of number of hops) to puller would not increase any further or even reduce due to shortest path routing. As a result, the delay of CHUM decreases when we take average over all users.

The average cost for COSMOS and CHUM are plotted in Figure 3(b). When the number of users increases, more peers collaborate to pull video data and the cost is shared among them. CHUM system does not have duplicate packets. Consequently, the average cost of CHUM drops much faster than that of COSMOS as $N$ increases. However, since only one peer pulls video data in CHUM network at the same time, the cost is distributed to a few of peers only. Some peers may not have chance to contribute and pull anything before leaving the system. The costs charged to peers are inconsistent. On the other hand, in COSMOS system, many peers collaboratively pull video descriptions simultaneously. Thus, telecommunication cost can be assigned to more peers, hence attaining higher fairness. This is illustrated in Figure 3(c) where we compare the cost fairness between CHUM and COSMOS. Clearly, CHUM in general has a 


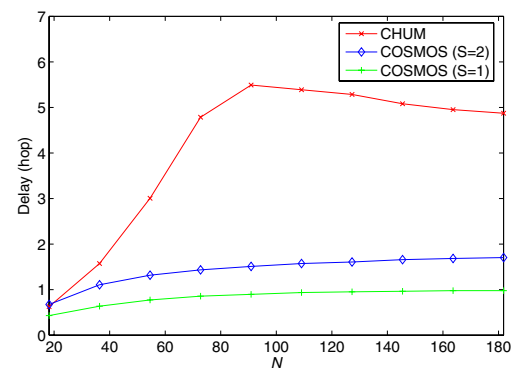

(a) Average Delay.

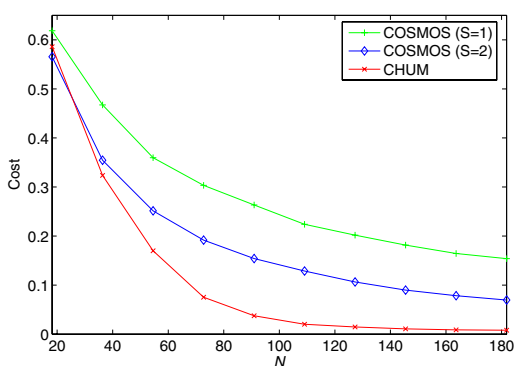

(b) Average Cost.

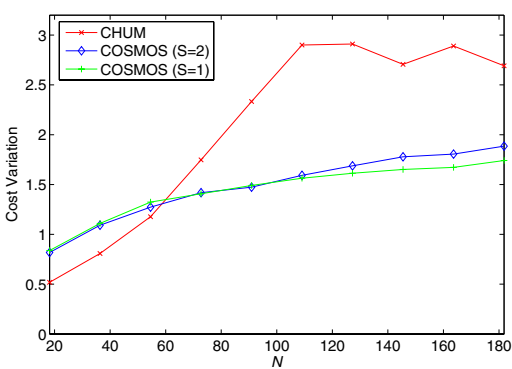

(c) Cost Variation.

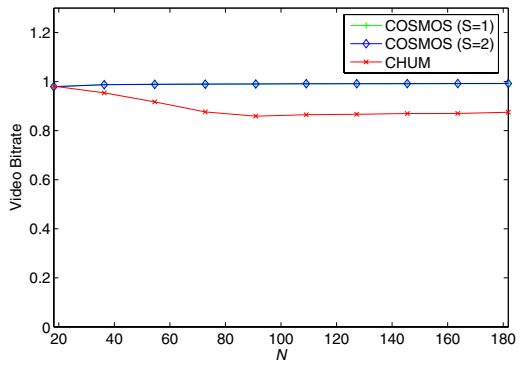

(d) Average Video Bitrate.

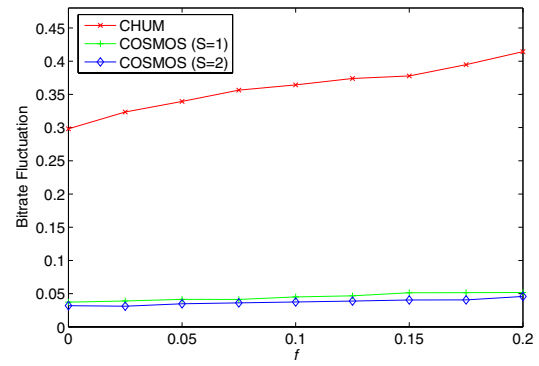

(e) Average Bitrate Fluctuation.

Fig. 3. Comparison of COSMOS and CHUM systems.

higher cost variation than COSMOS, as the telecommunication cost is more biased toward a few hosts.

Despite our protocol to reduce description duplication in the network, there are unavoidable description redundancy in the network. This is in fact an advantage as it leads to higher failure tolerance. Furthermore, the use of MDC minimizes the stream disruption when peer fails. The neighbors of the failure peer would experience the loss of one description only rather than the entire video clip as in CHUM. Hence, the video bitrates of peers can be kept rather steady. In CHUM, once a peer fails, other peers may suffer stream discontinuity, which affects the bitrate adversely. We show in Figure 3(d) the average bitrate received per node. Clearly, due to the above reasons, COSMOS achieves higher video bitrate than CHUM. We show in Figure 3(e) the bitrate variation as failure rate $f$ increases. Clearly, CHUM suffers from a larger bitrate fluctuation. The bitrate for COSMOS is more steady due to inheritance redundancy and MDC.

\section{CONCLUSION AND FUTURE WORK}

This paper proposes a scalable and cost-effective protocol called COSMOS (Collaborative Streaming among Mobiles) to distribute multimedia content to a group of mobile devices. COSMOS may incorporate with multiple description coding (MDC) for higher fault tolerance and stream stability. Each peer randomly selects and pulls an unavailable video description through a telecommunication link. It shares the description with its neighbors by broadcasting it so that its neighbors obtain more descriptions without increasing their streaming cost. Furthermore, peers take turns in pulling and hence the cost can be distributed more fairly. By controlling the broadcast scope, the delay of the system can be limited. Our simulation results show that COSMOS indeed achieves higher fault tolerance, more stable stream, and better fairness as compared with a previous scheme CHUM. We are currently studying the data confidentiality issue in COSMOS and associated key distribution mechanism. The incentive issue is also being investigated.

\section{REFERENCES}

[1] IEEE, "Wireless LAN Medium Access Control (MAC) and Physical Layer (PHY) specifications," in IEEE Standard 802.11, Sept. 1999.

[2] P. Bhagwat, "Bluetooth: technology for short-range wireless apps," IEEE Internet Computing, vol. 5, pp. 96-103, 2001.

[3] S. Kang and M. Mutka, "A mobile peer-to-peer approach for multimedia content sharing using 3G/WLAN dual mode channels," Wireless Communications and Mobile Computing, vol. 5, pp. 633-645, Sept. 2005.

[4] J. G. Apostolopoulos, "Error-resilient video compression through the use of multiple states," in Proceedings of IEEE ICIP, Sept. 2000, vol. 3, pp. 352-355.

[5] V. A. Vaishampayan and S. John, "Balanced interframe multiple description video compression," in Proceedings of ICIP, Oct. 1999, vol. 3, pp. 812-816.

[6] E. Yanmaz, O. Tonguz, S. Mishra, Hongyi Wu, and Chunming Qiao, "Efficient dynamic load balancing algorithms using iCAR systems: a generalized framework," in Proceedings of Vehicular Technology Conference. IEEE, Fall 2002, pp. 586-590.

[7] J.-C. Chen, S. Li, S.-H. Chan, and J.-Y. He, "WIANI: Wireless infrastructure and ad-hoc network integration," in Proceedings of IEEE International Conference on Communications (ICC), Korea, May 2005, IEEE, pp. 3623-3627. 